ISSN 0103-8478

\title{
Himenópteros parasitoides associados a ninfas de Triozoida limbata na cultura da goiabeira, em Ivinhema, MS, Brasil
}

\author{
Parasitoid Hymenoptera associated with Triozoida limbata nymphs in guava plantation, \\ at Ivinhema, MS, Brazil
}

\author{
Vera Alves de Sá ${ }^{I^{*}}$ Marcos Gino Fernandes ${ }^{I}$
}

\section{- NOTA -}

\section{RESUMO}

O Brasil tem excelentes condições climáticas para a exploração comercial da goiabeira Psidium guajava L. (Myrtaceae); porém, em todo o país, a cultura é atacada por insetos-praga, sendo Triozoida limbata Enderlein, 1918 (Hemiptera: Triozidae) uma das pragas dessa cultura. Como o controle químico dessa praga é muito oneroso e, muitas vezes, indesejável, a utilização de parasitoides é uma excelente alternativa, pois pode atuar eficientemente na regulação das populações dos seus hospedeiros. Assim, este trabalho objetivou identificar as espécies de parasitoides ocorrentes e seus indices de parasitismo natural em $\mathbf{T}$. limbata no cultivo da goiabeira, em Ivinhema, MS. Foram realizadas coletas quinzenais de março a outubro de 2010, sendo coletados dez ramos terminais das plantas com ninfas de T. limbata, por coleta, sempre no início da manhã. Estes ramos tinham suas bases enroladas em algodão hidrófilo, introduzidas em frascos de vidro $(5 \mathrm{~mL})$ com água e acondicionados em copos transparentes de acrílico justapostos, e assim mantidos até que ocorresse a emergência dos parasitoides adultos. Para identificação, foram calculadas a porcentagem e o indice de parasitismo natural. Do total de 349 espécimes de parasitoides encontrados, 91,11\% foi Psyllaephagus trioziphagus Howard, 1885 (Hymenoptera: Encyrtidae), com 20,96\% de parasitismo natural, e 8,89\% foram representantes do gênero Signiphora, com 2,04\% de parasitismo natural.

Palavras-chave: controle biológico, fruticultura, inseto sugador.

\section{ABSTRACT}

Brazil has an excellent climate for commercial use of guava Psidium guajava L. (Myrtaceae). However, guava crops throughout Brazil are severely attacked by the insect Triozoida limbata Enderlein, 1918 (Hemiptera: Triozidae), one of the pest of guava crops. The control of this pest with chemicals is costly and often undesirable, whereas parasitoids are a great alternative because they can efficiently regulate their host populations. This study aimed to identify the parasitoid species in T. limbata occurring in a guava plantation and their rates of parasitism, in Ivinhema, MS, Brazil. Data were collected every two weeks early in the morning from March to October 2010, collecting 10 terminal branches from plants with $\mathrm{T}$. limbata nymphs. The base of the branches were wrapped in cotton wool, placed in 5-mL glass vials with water, packed in transparent acrylic glasses, juxtaposed, and maintained until adult parasitoids emerged. These samples were used to calculate the percentage and index of parasitism. From the 349 parasitoid specimens found, 91.11\% were Psyllaephagus trioziphagus Howard, 1885 (Hymenoptera: Encyrtidae) with $20.96 \%$ of parasitism, whereas $8.89 \%$ were from the genus Signiphora with $2.04 \%$ of parasitism.

Key words: biological control, horticulture, sucking insect.

O Brasil se destaca no cenário mundial entre os principais produtores de goiaba Psidium guajava L. (Myrtaceae), juntamente com a Índia, Paquistão e México. Essa cultura, durante seu desenvolvimento, é atacada por diversos insetos-praga que provocam diferentes tipos de injúrias (SÁ, 2011). Estes insetos são um dos principais obstáculos ao seu cultivo, pois eles são capazes de reduzir severamente a produção e a qualidade dos frutos (NDANKEU et al., 2011). Entre os insetos que atacam a goiabeira, pode-se citar Triozoida limbata Enderlein, 1918 (Hemiptera: Triozidae) (MAES et al., 1993), que é uma das pragaschave dessa frutífera (COLOMBI \& GALLI, 2009).

As ninfas possuem formato achatado, são de coloração rósea, apresentam-se recobertas por excreção de cera de coloração esbranquiçada; quando as ninfas atingem o terceiro ínstar, movemse para as bordas das folhas, quando sugam a seiva

IPrograma de Pós-graduação em Entomologia e Conservação da Biodiversidade, Faculdade Ciências Biológicas e Ambientais, Universidade Federal da Grande Dourados (UFGD), 79804-970, Dourados, MS, Brasil.E-mail: veraalves_bio@ yahoo.com.br.*Autor para correspondência. Recebido 12.03.14 Aprovado 19.06.14 Devolvido pelo autor 09.9.14 CR-2014-0382.R1 
(SEMEÃO et al., 2012a). Injetam toxinas que enrola os bordos do limbo foliar, tornando-as deformadas, com coloração avermelhada e, posteriormente, aspecto necrosado (NDANKEU et al., 2011). Os enrolamentos dos bordos foliares protegem as ninfas até se tornarem adultas, tornando baixa a eficiência das intervenções de controle, tais como inseticidas. Consequentemente, inimigos naturais que se adaptam a esta situação apresentam condições que podem superar essa barreira e são componentes do Manejo Integrado de Pragas (MIP) e podem afetar a dinâmica populacional de $\boldsymbol{T}$. limbata (SEMEÃO et al., 2012b).

Um bom exemplo de inimigos naturais são as espécies de himenópteros parasitoides, que são consideradas componentes importantes na manutenção do balanço ecológico e promotores da diversidade de outros organismos. Devido à sua capacidade de regular a população de outros insetos, muitas espécies têm sido utilizadas com sucesso no controle biológico (SÁ \& FERNANDES, 2012).

Desta forma, este trabalho objetivou identificar as espécies de parasitoides ocorrentes e seus índices de parasitismo natural em T. limbata, no cultivo da goiabeira, em Ivinhema, MS.

O estudo foi desenvolvido em um pomar de goiabeiras 'Pedro Sato', com 550 plantas, de três anos e meio de idade, espaçamento entre plantas de $5 \times 7 \mathrm{~m}$, sem uso de agrotóxicos, no município de Ivinhema MS, Brasil (22 $12^{\circ}$ '42,55” S; 53 48' 38,68”'O e $341 \mathrm{~m}$ de altitude).

Foram coletados, quinzenalmente, no período da manhã, de março a outubro de 2010, 10 ramos terminais, com seis centímetros de comprimento, de goiabeiras com presença de ninfas de T. limbata. As bases dos ramos foram enroladas em algodão hidrófilo, introduzidas em frascos de vidro $(5 \mathrm{~mL})$ com água. Esses ramos foram, então, acondicionados no interior de copos transparentes de acrílico justapostos. Os copos foram mantidos climatizados a $25 \pm 2^{\circ} \mathrm{C}$ e fotofase de 12 horas, até a emergência dos parasitoides ou, então, até o secamento total dos ramos. Os ramos no interior dos copos eram observados diariamente para a coleta dos parasitoides adultos. Os parasitoides coletados foram depositados em eppendorfs contendo álcool etílico $70 \%$ e, posteriormente, identificados pelo Dr. Valmir Antonio Costa, do Centro Experimental do Instituto Biológico, em Campinas - SP.

$\mathrm{O}$ índice de parasitismo natural foi calculado de acordo com BITTENCOURT et al. (2012). Os espécimes coletados foram depositados na coleção de Insetos Entomófagos "Oscar Monte", no laboratório de Controle Biológico do Instituto Biológico, em Campinas - SP.
Dos ramos coletados, emergiram 349 espécimes de parasitoides, sendo que 91,11\% foi Psyllaephagus trioziphagus (Howard, 1885) (Hymenoptera: Encyrtidae), atingindo um índice de parasitismo natural de $20,96 \%$. O restante dos parasitoides obtidos $(8,89 \%)$ foi representante do gênero Signiphora, com o índice de parasitismo de 2,04\%.

O parasitoide $\boldsymbol{P}$. trioziphagus, até o momento, apenas foi encontrado em poucas espécies de Triozidae. A maioria das espécies desse gênero são parasitoides primários de Hemiptera, e várias espécies estão sendo utilizadas em programas de controle biológico de Psylloidea. Como na Califórnia, EUA e na Nova Zelândia, no controle de Glycaspis brimblecombei Moore (Hemiptera: Psylloidea), praga na cultura do Eucalyptus sp. (DANNE et al., 2005; BERRY, 2007), e na Austrália no controle de Acizzia solanicola Kent \& Taylor, 2010 (Hemiptera: Triozidae), praga em plantações de berinjelas (KENT \& TAYLOR, 2010).

$\mathrm{O}$ gênero Signiphora é considerado um parasitoide secundário, numeroso na região Neotropical e, quando encontrado em alto número, pode interferir negativamente no controle biológico de uma praga (MELO, 2009), o que não foi observado no pomar em estudo.

Devido à importância do controle natural, existe uma necessidade de estudos que determinem o papel de tais espécies na dinâmica das populações de insetos-praga em diferentes agroecossistemas (SEMEÃO et al., 2012a). Os resultados desse e de outros estudos (MELO, 2009; SEMEÃO et al., 2012a) indicam que $\boldsymbol{P}$. trioziphagus é um parasitoide que merece ser alvo de futuros estudos, visando a conhecer as características biológicas, técnicas de multiplicação em laboratório e posterior liberação em campo, sendo que contribuirá para manter as populações da praga alvo em níveis economicamente aceitáveis, conforme apontado por SEMEÃO et al. (2012b).

\section{AGRADECIMENTOS}

Ao Conselho Nacional de Desenvolvimento Científico e Tecnológico (CNPq), pela concessão de bolsa de Mestrado ao primeiro autor. Ao Dr. Valmir Antonio Costa, do Instituto Biológico de São Paulo, pela identificação dos parasitoides.

\section{REFERÊNCIAS}

BERRY, J.A. Key to the New Zealand species of Psyllaephagus Ashmead (Hymenoptera: Encyrtidae) with descriptions of three new species and a new record of the psyllid hyperparasitoid Coccidoctonus psyllae Riek (Hymenoptera: Encyrtidae). Australian Journal of Entomology, Brisbane, v.46, p.99-105, 2007. Disponível em: <http://onlinelibrary.wiley.com/doi/10.1111/ j.1440-6055.2007.00575.x/pdf $>$. Acesso em: 15 fev. 2013. doi:10.1111/j.1440-6055.2007.00575.x. 
BITTENCOURT, M.A.L. et al. Parasitóides (Braconidae) associados à Anastrepha (Tephritidae) em frutos hospedeiros do Litoral Sul da Bahia. Ciência Agronômica, Fortaleza, v.43, n.4, p.811-815, 2012. Disponível em: <http://www.scielo.br/scielo. php?script=sci_arttext\&pid=S1806-66902012000400024\&lng= en\&nrm=iso\&tlng=pt>. Acesso em: 05 jan. 2014. doi: 10.1590/ S1806-66902012000400024.

COLOMBI, C.A.; GALLI, J.C. Dinâmica populacional e evolução de dano de Triozoida limbata (Hemiptera: Psillydae) em goiabeira, em Jaboticabal, SP. Ciência e Agrotecnologia, Lavras, v.33, p.412-416, 2009. Disponível em: <http://www.scielo.br/scielo. php?pid=S 1413-70542009000200008\&script $=$ sci_arttext $>$ Acessoem: 10 jan. 2013. doi: 10.1590/S1413-70542009000200008.

DAANE, K.M. et al. The biology of Psyllaephagus bliteus Riek (Hymenoptera: Encyrtidae), a parasitoid of the red gum lerp psyllid (Hemiptera: Psylloidea). Biological Control, San Diego, v.32, p.228-235, 2005. Disponível em: 〈http://www.sciencedirect. com/science/article/pii/S1049964404001896>. Acesso em: $11 \mathrm{fev}$. 2011. doi:10.1016/j.biocontrol.2004.09.015.

KENT, D.; TAYLOR, G. Two new species of Acizzia Crawford (Hemiptera: Psyllidae) from the Solanaceae with a potential new economic pest of eggplant, Solanum melongena. Australian Journal of Entomology, Brisbane, v.49, p.73-81, 2010. Disponível em: <http://onlinelibrary.wiley.com/doi/10.1111/ j.1440-6055.2009.00739.x/pdf $>$. Acesso em: 10 nov. 2011. doi: 10.1111/j.1440-6055.2009.00739.x.

MAES, J. et al. Catalogo de los Psylloidea (Hemiptera) de Nicaragua. Revista Nicaraguense de Entomologia, León, v.26, p.1-6, 1993. Disponível em: <http://www.bio-nica.info/ RevNicaEntomo/RevNicaEntomo.htm>. Acesso em: 15 mar. 2010 .

MELO, G. Dinâmica populacional e inimigos naturais de Triozoida limbata (Hemiptera: Triozidae) e diversidade de famílias de himenópteros parasitoides em pomar convencional e orgânico de goiaba na região de Campinas, SP. 2009. 66f.
Dissertação (Mestrado em Sanidade Segurança Alimentar e Ambiental no Agronegócio) - Instituto Biológico.

NDANKEU, Y.P.M. et al. Biodiversity of jumping plant-lice of the Psyllidae family (Hemiptera: Psylloidea) from the South Region of Cameroon: faunistics, phenology and host plants. Journal of Entomology, Brisbane, v.8, p.123-138, 2011. Disponível em: <http://core.kmi.open.ac.uk/display/968259>. Acesso em: 15 fev. 2012. doi: 10.3923/je.2011. 123.138.

SÁ, V.A. Comportamento de acasalamento, níveis de infestação e parasitismo de Triozoida limbata Enderlein, 1918 (Hemiptera: Triozidae) em Psidium guajava L. (Myrtaceae). 2011. 53f. Dissertação (Mestrado em Entomologia e Conservação da Biodiversidade) - Universidade Federal da Grande Dourados.

SÁ, V.A.; FERNANDES, M.G. Himenópteros parasitoides de Triozoida limbata Enderlein, 1918 (Hemiptera: Triozidae) em Psidium guajava L. (Myrtaceae). In: CONGRESSO BRASILEIRO DE ENTOMOLOGIA, 2012, Curitiba, PR. Anais... Curitiba: Sociedade Entomológica do Brasil, 2012. 2437p. p.1372.

SEMEÃO, A.A. et al. Life tables for the guava psyllid Triozoida limbata in southeastern Brazil. BioControl, Dordrecht, v.1, p.110, 2012a. Disponível em: <http://link.springer.com/content/ pdf/10.1007/s10526-012-9458-y.pdf >. Acesso em: 10 jan 2013. doi: 10.1007/s10526-012-9458-y.

SEMEÃO, A.A. et al. Seasonal variation of natural mortality factors of the guava psyllid Triozoida limbata. Bulletin of Entomological Research, v.1, p.1-11, 2012b. Disponível em: <http://www.ncbi. nlm.nih.gov/pubmed/22677036>. Acesso em: 11 jan. 2013. doi: $10.1017 /$ S0007485312000338.

TAMESSE, J.L. Key for identification of the Hymenopteran parasitoids of the African citrus psylla Trioza erytreae Del Guercio (Hemiptera: Triozidae) in Cameroon. African Journal of Agricultural Research, Nairobi, v.4, p.85-91, 2009. Disponível em: <http://www.academicjournals.org/ajar/pdf/pdf\%202009/Feb/ Tamesse.pdf $>$. Acesso em: 22 nov. 2010. 